\title{
Emprego da análise AMMI na avaliação da estabilidade produtiva em soja ${ }^{(1)}$
}

\author{
Adriano Borges de Oliveira( ${ }^{(2)}$, João Batista Duarte ${ }^{(3)}$ e José Baldin Pinheiro ${ }^{(3)}$ \\ Resumo - O objetivo deste trabalho foi avaliar a influência da interação de genótipos com ambientes \\ (GxA) na produtividade de grãos de um conjunto de linhagens de soja (Glycine max L.). Foram utiliza- \\ dos dados de 11 experimentos (ambientes) realizados no Estado de Goiás. Em cada experimento foram \\ avaliados 18 genótipos, sendo quatro cultivares comerciais como testemunhas. O método de análise da \\ interação foi o procedimento AMMI (modelo de efeitos principais aditivos e interação multiplicativa). \\ $\mathrm{O}$ padrão significativo das interações GxA foi captado apenas pelo primeiro eixo principal AMMI, o \\ qual explicou 36\% da soma de quadrados GxA original, sugerindo contaminação da matriz de interações \\ clássica por ruídos que prejudicam a qualidade das predições de respostas fenotípicas obtidas pelos \\ métodos tradicionais. Quanto à estabilidade de comportamento, a maioria das linhagens experimentais \\ destacou-se (com menores interações com ambientes) em relação às cultivares testemunhas. Estas, no \\ entanto, foram relativamente mais produtivas, sobretudo a cultivar Conquista. Entre as novas linha- \\ gens, os genótipos L-16, L-13 e L-14 mostraram ser os mais promissores para fins de recomendação \\ como cultivares.
}

Termos para indexação: Glycine max, progênie, interação genótipo-ambiente, adaptação, biplot.

\section{Application of AMMI analysis in the assessment of yield stability in soybean}

\begin{abstract}
The objective of this work was to evaluate the influence of the genotype environment (GE) interaction on the grain yield of a soybean (Glycine max L.) lines group. Yield data from 11 trials (environments) conducted in the State of Goiás, Brazil were used. In each trial 18 genotypes were tested, from which four were commercial cultivars as checks. The statistical method was the AMMI analysis (additive main effect and multiplicative interaction analysis). A significant GE interaction pattern was captured only for the first principal AMMI axis, which explained 36\% of the original square sum of the GE interaction, suggesting contamination of the classic GE interaction matrix by noise arising from unpredictable factors, assuring that AMMI analysis provides a better prediction of phenotypic responses than traditional methodologies. About yield stability, most experimental lines outstands (with low GE interaction) over checks. However, the checks, mainly the cultivar Conquista, showed higher yield averages. Among the experimental lines, the genotypes L-16, L-13 and L-14 appeared to be the most promising for cultivars recommendation.
\end{abstract}

Index terms: Glycine max, progeny, genotype environment interaction, adaptation, biplot.

\section{Introdução}

As interações de genótipos com ambientes (GxA) trazem aos melhoristas dificuldades na identificação

(1)Aceito para publicação em 17 de dezembro de 2002.

Extraído da dissertação de mestrado apresentada pelo primeiro autor à Universidade Federal de Goiás (UFG), Goiânia, GO. Financiada pela Capes.

${ }^{(2)}$ Fundação de Desenvolvimento, Assistência Técnica e Extensão Rural de Goiás, Av. Anhanguera Qd. 117-B Lt. 03 no 122, Setor Universitário, CEP 74612-010 Goiânia, GO. E-mail: adboliveira@bol.com.br

${ }^{(3)}$ UFG, Escola de Agronomia e Engenharia de Alimentos, Caixa Postal 131, CEP 74001-970 Goiânia, GO. E-mail: jbduarte@uol.com.br, baldin@agro.ufg.br de genótipos superiores, seja por ocasião da seleção, seja no momento da recomendação de cultivares. A presença dessas interações indica que o comportamento relativo dos genótipos nos testes depende, fundamentalmente, das condições ambientais a que estão submetidos. Desta forma, a resposta fenotípica de qualquer genótipo em relação a outros poderá ser inconsistente, o que se manifesta pela alteração da posição relativa dos genótipos de um ambiente para outro, ou em alterações na magnitude das diferenças absolutas entre seus fenótipos, sem que a sua ordem seja alterada. Em síntese, essas respostas genotípicas diferenciadas a ambientes distintos são coletivamente chamadas de interação de genótipos com ambientes (Kang, 1998). 
A cultura da soja é comumente submetida a inúmeras variações ambientais e a interação GxA assume papel importante na manifestação fenotípica. Por isso, deve ser estimada buscando-se avaliar sua importância na recomendação de cultivares e na condução de programas de melhoramento genético. Sediyama et al. (1990) afirmam que estudos criteriosos de adaptabilidade e estabilidade de produção em soja devem ser realizados para garantir maior segurança às recomendações de cultivares.

Nesse sentido, a aplicação de procedimentos de regressão linear tem alcançado grande popularidade (Eberhart \& Russell, 1966; Cruz et al., 1989; Silva, 1995). Alguns estudos, porém, têm mostrado que uma parte razoável dos genótipos não tem seus comportamentos satisfatoriamente explicados por esses métodos (Zobel et al., 1988; Duarte \& Zimmerman, 1994).

Um método de aplicação mais recente, que também permite inferências dessa natureza, é a chamada análise AMMI (additive main effects and multiplicative interaction analysis), que significa modelo de efeitos principais aditivos e interação multiplicativa. Este método combina técnicas estatísticas, como a análise de variância e a análise de componentes principais, para ajustar, respectivamente, os efeitos principais (genótipos e ambientes) e os efeitos da interação GxA (Mandel, 1971; Kempton, 1984; Zobel et al., 1988). A análise AMMI pode ajudar tanto na identificação de genótipos de alta produtividade e largamente adaptados como na realização do chamado zoneamento agronômico, com fins de recomendação regionalizada e de seleção de locais de teste (Gauch \& Zobel, 1996). Zobel et al. (1988) enumeram algumas vantagens desse método: permite uma análise mais detalhada da interação GxA; garante a seleção de genótipos, capitalizando suas interações positivas com os ambientes; propicia estimativas mais precisas das respostas genotípicas; possibilita fácil interpretação gráfica dos resultados, nos chamados biplots (representação gráfica simultânea dos genótipos e ambientes).

No que se refere ao detalhamento da interação GxA, essa análise o faz via decomposição da soma de quadrados original $\left(\mathrm{SQ}_{\mathrm{GxA}}\right)$, em uma porção denominada padrão e noutra chamada ruído.
A primeira possibilita a identificação de fatores ambientais e genotípicos mais diretamente relacionados à interação, sendo obtida após o descarte de ruídos adicionais ao erro experimental. Isso proporciona uma melhoria na capacidade preditiva das respostas fenotípicas. Embora ainda pouco utilizada no Brasil, sua aplicação já se faz presente em espécies cultivadas, como o trigo (Kempton, 1984; Crossa et al., 1991), o milho (Hirotsu, 1983) e a soja (Gauch, 1988; Zobel et al., 1988).

O objetivo deste trabalho foi avaliar a influência da interação de genótipos com ambientes (GxA) na produtividade de grãos de um conjunto de linhagens de soja.

\section{Material e Métodos}

Os dados foram provenientes de experimentos de avaliação de linhagens de soja desenvolvidas na Escola de Agronomia e Engenharia de Alimentos da Universidade Federal de Goiás. Essas linhagens pertencem ao grupo de maturação precoce, estando na geração $\mathrm{F}_{10}$ de autofecundação (elevado nível de homozigose). Como testemunhas, foram utilizadas as cultivares comerciais FT-2000, Emgopa 315, MSOY-8001 e Conquista. Foram avaliados 18 genótipos: 14 linhagens experimentais e 4 cultivares testemunhas. Os experimentos foram realizados no ano agrícola 2000/2001, em três municípios goianos (Goiânia, Itumbiara e Jataí) e em quatro épocas de semeadura (28/nov., 4/dez., 11/dez. e 18/dez.). Os locais, além de suas diversidades climáticas, apresentaram variações em fertilidade do solo (Tabela 1).

Os experimentos foram instalados no delineamento de blocos casualizados, com três repetições. As parcelas foram constituídas de quatro fileiras de 5,0 m de comprimento, espaçadas em 0,5 m. Como parcela útil, foram considerados os 4,0 m centrais das duas fileiras internas, desprezando-se as duas laterais e $0,50 \mathrm{~m}$ nas extremidades das duas centrais. A densidade de semeadura foi de 20 sementes por metro. As práticas culturais foram realizadas conforme Embrapa (2000).

A colheita foi realizada quando $90 \%$ das vagens das plantas da parcela encontravam-se maduras. Os grãos de cada parcela foram acondicionados em sacos de papel, secados ao sol e pesados.

Para avaliação estatística da variabilidade genética entre os tratamentos (genótipos) e da precisão experimental, foi realizada análise de variância de cada ambiente (análises individuais). Uma vez detectada diferenciação entre os tratamentos, realizou-se análise conjunta de variância 
(Ramalho et al., 2000), a qual foi implementada mesmo não tendo havido homogeneidade entre as variâncias residuais $\left(\mathrm{QM}_{\mathrm{R}}\right.$ 's). Assim, procedeu-se ao ajuste dos graus de liberdade do erro médio e da interação GxA, segundo o método de Cochran (1954). Somente após esses ajustes foram feitas as interpretações relativas às significâncias do teste F.

Uma vez constatada a presença de interação GxA (teste $\mathrm{F}$ significativo), procedeu-se à análise de estabilidade, a qual permite mensurar a adaptação e a estabilidade produtiva de cada linhagem sob teste. Tal avaliação foi feita pelo modelo AMMI de análise:

$\mathrm{Y}_{\mathrm{ij}}=\mu+\mathrm{g}_{\mathrm{i}}+\mathrm{a}_{\mathrm{j}}+\sum_{\mathrm{k}=1}^{\mathrm{n}} \lambda_{\mathrm{k}} \gamma_{\mathrm{ik}} \alpha_{\mathrm{jk}}+\rho_{\mathrm{ij}}+\overline{\mathrm{e}}_{\mathrm{ij}}$,

em que: $\mathrm{Y}_{\mathrm{ij}}$ é a resposta média do genótipo i $(\mathrm{i}=1,2, \ldots$, G genótipos) no ambiente $\mathrm{j}(\mathrm{j}=1,2, \ldots, \mathrm{A}$ ambientes $) ; \mu$ é a média geral dos ensaios; $g_{i}$ é o efeito fixo do genótipo i;

$\mathrm{a}_{\mathrm{j}}$ é o efeito fixo do ambiente $\mathrm{j} ; \lambda_{\mathrm{k}}$ é o k-ésimo valor singular (escalar) da matriz de interações original (denotada por

$\mathrm{GA}) ; \gamma_{\mathrm{ik}}$ é o elemento correspondente ao i-ésimo genótipo no k-ésimo vetor singular coluna da matriz GA; $\alpha_{j k}$ é o elemento correspondente ao j-ésimo ambiente no k-ésimo vetor singular linha da matriz GA; $\rho_{\mathrm{ij}}$ é o ruído associado ao termo $(\mathrm{ga})_{\mathrm{ij}}$ da interação clássica do genótipo i com o ambiente $\mathrm{j}$; $\overline{\mathrm{e}}_{\mathrm{ij}}$ é o erro experimental médio.

Nota-se, portanto, que o termo $(\mathrm{ga})_{\mathrm{ij}}$ de um modelo de análise conjunta tradicional é modelado por:

$(\mathrm{ga})_{\mathrm{ij}}=\sum_{\mathrm{k}=1}^{\mathrm{n}} \lambda_{\mathrm{k}} \gamma_{\mathrm{ik}} \alpha_{\mathrm{jk}}+\rho_{\mathrm{ij}}$,

sendo:

$\rho_{\mathrm{ij}}=\sum_{\mathrm{k}=\mathrm{n}+1}^{\mathrm{p}} \lambda_{\mathrm{k}} \gamma_{\mathrm{ik}} \alpha_{\mathrm{jk}}$,

em que p é o posto da matriz GA. Os termos $\lambda_{\mathrm{k}}, \gamma_{\mathrm{ik}}$ e $\alpha_{\mathrm{jk}}$ resultam da chamada decomposição por valores singulares (DVS) da matriz de interações clássica: $\mathrm{GA}_{(\mathrm{gxa})}=\left[(\hat{\mathrm{ga}})_{\mathrm{ij}}\right]$, com: $\left(\stackrel{\hat{g a a}}{\mathrm{ijj}}=\mathrm{Y}_{\mathrm{ij}}-\overline{\mathrm{Y}}_{\mathrm{i} .}-\overline{\mathrm{Y}}_{. \mathrm{j}}+\overline{\mathrm{Y}}_{. .}\right.$,

em que: $\bar{Y}_{\mathrm{i}}$ é a média do genótipo $\mathrm{i} ; \overline{\mathrm{Y}}_{\mathrm{j}}$ é a média do ambiente $\mathrm{j}$; e $\overline{\mathrm{Y}}$ é a média geral dos ensaios. A obtenção da DVS de uma matriz, nesse tipo de aplicação, é descrita por Duarte \& Vencovsky (1999). Esses autores comentaram que, no modelo AMMI, o efeito da interação do genótipo i com o ambiente j é descrito como uma soma de " $n$ " parcelas, cada uma resultante da multiplicação de $\lambda_{\mathrm{k}}$, expresso na mesma unidade de $\mathrm{Y}_{\mathrm{ij}}$, por efeitos de escala (adimensionais) genotípico $\left(\gamma_{\mathrm{ik}}\right)$ e ambiental $\left(\alpha_{\mathrm{jk}}\right)$. O termo $\lambda_{\mathrm{k}}$ traz uma informação relativa à variação resultante da interação GxA, na k-ésima dessas parcelas. Como numa análise de componentes principais (ACP), essas sucessivas parcelas captam porções cada vez menores da variação presente na matriz GA $\left(\lambda_{1}^{2} \geq \lambda_{2}^{2} \geq \ldots \geq \lambda_{p}{ }^{2}\right)$, isto é, a soma de quadrados $\mathrm{SQ}_{\mathrm{GxA}}$ tradicional. Assim, a análise busca recuperar uma parcela determinística da matriz denominada padrão, descartando-se outra denominada ruído $\left(\rho_{\mathrm{ij}}\right)$.

A definição do número "n" de eixos principais retidos baseou-se no teste F de Gollob (1968) e de Cornelius et al. (1992), bem como no procedimento de avaliação preditiva por validação cruzada, proposto por Gauch (1988). Todos esses métodos estão descritos em Duarte \& Vencovsky (1999), incluindo-se uma rotina computacional em linguagem SAS/IML para implementação das análises.

As coordenadas de genótipos e de ambientes nos eixos principais de interação (IPCA) são representadas em um gráfico denominado biplot (Gabriel, 1971), que permite descrever a estabilidade e a adaptabilidade dos genótipos aos ambientes de teste.

\section{Resultados e Discussão}

A interação dos genótipos com os ambientes mostrou-se altamente significativa (Tabela 2), ou seja, os genótipos sofreram influências diferenciadas dos ambientes, o que dificulta a recomendação de cultivares para a região abrangida pelo estudo. É necessário estudo mais detalhado dessa interação, de modo

Tabela 1. Características médias das três localidades do Estado de Goiás onde se realizaram os experimentos de avaliação de genótipos de soja.

\begin{tabular}{lccccccc}
\hline Local & $\begin{array}{c}\text { Altitude } \\
(\mathrm{m})\end{array}$ & Latitude & $\begin{array}{c}\text { Temp. máx. } \\
\left({ }^{\circ} \mathrm{C}\right)\end{array}$ & $\begin{array}{c}\text { Temp. mín. } \\
\left({ }^{\circ} \mathrm{C}\right)\end{array}$ & $\begin{array}{c}\mathrm{PT}^{(1)} \\
(\mathrm{mm})\end{array}$ & $\begin{array}{c}\mathrm{PPR}^{(2)} \\
(\mathrm{mm})\end{array}$ & $\begin{array}{c}\mathrm{V}^{(3)} \\
(\%)\end{array}$ \\
\hline Itumbiara & 449 & $18^{\circ} 25^{\prime}$ & 32 & 20 & 685,53 & 406,35 & 44 \\
Goiânia & 730 & $16^{\circ} 41^{\prime}$ & 31 & 23 & 851,30 & 472,20 & 37 \\
Jataí & 708 & $17^{\circ} 53^{\prime}$ & 31 & 19 & 837,93 & 423,65 & 36 \\
\hline
\end{tabular}

${ }^{(1)}$ Precipitação pluvial total no período da semeadura à maturação fisiológica. ${ }^{(2)}$ Precipitação no período reprodutivo da cultura (R1 a R7). ${ }^{(3)}$ Saturação de bases do solo. 
que ela possa ser controlada, e não interfira negativamente na recomendação.

Em concordância com o modelo AMMI, a interação GxA original pôde ser decomposta em dez componentes (posto da matriz GA), dos quais apenas o primeiro termo (IPCA1) foi julgado como altamente significativo. Os resíduos do AMMI1 mostraram-se não-significativos, a 1\% de probabilidade, confirmando a seleção de AMMI1. Este modelo explicou aproximadamente $36 \%$ da $\mathrm{SQ}_{\mathrm{GxA}}$, embora usando menos de $20 \%$ dos graus de liberdade originais (Tabela 3).

A seleção do modelo AMMI1 também foi confirmada pelo procedimento de avaliação preditiva. Por este critério, o modelo AMMI1 resultou em menor valor de diferença preditiva média (RMSPD) em $44 \%$ das análises, contra $23 \%$ e $22 \%$ dos modelos AMMI0 e AMMI2, respectivamente. A estatística RMSPD reflete o desvio das predições do modelo para cada combinação de genótipo e ambiente em relação a dados de validação (observações excluídas do ajustamento do modelo, por sorteio). Assim, num elevado número de análises a preferência deve

Tabela 2. Análise conjunta de variância da produtividade de 18 genótipos de soja testados em 11 ambientes no Estado de Goiás, em 2000/2001.

\begin{tabular}{lcrrrc}
\hline Fonte de variação & GL & \multicolumn{1}{c}{ SQ } & \multicolumn{1}{c}{ QM } & \multicolumn{1}{c}{ F } & Pr > F \\
\hline Bloco/ambiente & 22 & $6.606 .873,00$ & $300.312,41$ & 2,37 & 0,0007 \\
Ambiente (A) & 10 & $76.247 .026,17$ & $7.624 .702,62$ & 60,23 & 0,0001 \\
Genótipo (G) & 17 & $12.134 .431,90$ & $713.790,11$ & 5,64 & 0,0001 \\
GxA & $151^{(1)}$ & $33.736 .948,33$ & $223.423,50$ & 1,76 & 0,0001 \\
Erro & $216^{(1)}$ & - & $126.598,90$ & - & - \\
\hline
\end{tabular}

(1) Valores ajustados pelo método Cochran (1954), em razão da heterogeneidade de QM's residuais.

Tabela 3. Análise de variância com desdobramento da interação original GxA pelo modelo AMMI, em nível de médias $^{(1)}$

\begin{tabular}{lccrcc}
\hline Fonte de variação & GL & SQ & QM & F & Pr > F \\
\hline Interação GxA & $151^{(2)}$ & $12.891 .459,05$ & $85.373,90$ & 1,76 & 0,0006 \\
IPCA1 (padrão) & 26 & $4.715 .627,11$ & $181.370,27$ & 3,75 & 0,0000 \\
Resíduo AMMI1 (ruído) & 125 & $8.175 .831,94$ & $65.406,66$ & 1,35 & 0,0273 \\
IPCA2 & 24 & $3.140 .704,47$ & $130.862,69$ & 2,71 & 0,0001 \\
Resíduo AMMI2 & 101 & $5.035 .127,47$ & $49.852,75$ & 1,03 & 0,4231 \\
Erro médio/r & $216^{(2)}$ & - & $48.375,58$ & - & - \\
\hline
\end{tabular}

${ }^{(1)} \mathrm{O}$ número de repetições foi 2,617 (média harmônica, em razão do desbalanceamento). (2)Valores ajustados pelo método Cochran (1954), em razão da heterogeneidade de QM's residuais. recair sobre o modelo cuja diferença preditiva média seja menor num maior número de análises.

Um aspecto metodológico observado foi que, embora Crossa et al. (1991) recomendem cerca de dez ciclos de validação cruzada para esse procedimento, verificou-se que tal número pode não ser suficiente. Nesse sentido, Piepho (1994) sugere até mil randomizações. No presente trabalho, entretanto, a realização de cem randomizações permitiu atestar a superioridade relativa do modelo AMMI1.

Nesse sentido, Piepho (1995) argumenta que o principal objetivo dos testes estatísticos disponíveis para esse fim é determinar a quantidade de termos multiplicativos não-nulos. Entretanto, isso não é o mesmo que buscar um número ótimo de termos para um modelo preditivo, como usualmente faz a validação cruzada. Ademais, um modelo preditivo geralmente tem menor número de termos do que o número julgado significativo por um teste. Assim, uma estratégia no sentido de selecionar um modelo preditivo é aumentar o rigor dos testes, reduzindo o seu nível de significância. Por isso, no presente trabalho, os testes foram utilizados a $1 \%$ de probabilidade, o que implica concordância dos testes pela seleção do modelo AMMI1.

Com respeito ao nível de significância, vale salientar que, com a utilização de $1 \%$ em vez de $5 \%$, reduz-se a probabilidade de ocorrer o erro tipo I, isto é, de aceitar um modelo AMMI com maior número de eixos, quando na verdade o modelo correto é menos parametrizado. Porém, aumenta-se a probabilidade de ocorrer o erro tipo II, ou seja, de aceitar um modelo AMMI com menor número de eixos mas cujo modelo correto seja de fato mais parametrizado. Uma das premissas da análise AMMI é a de que nos primeiros eixos concentra-se maior porcentagem de "padrão". Assim, à medida que se eleva o número de eixos selecionados, aumenta-se a porcentagem de "ruído", reduzindo o poder de predição da análise AMMI. Gauch (1988) e Gauch \& Zobel (1996) mostraram que a inclusão excessiva de termos multiplicativos pode reduzir seriamente a acurácia da análise. Cornelius et al. (1992) também destacaram a importância de se controlar o erro tipo I, sugerindo testes de seleção de modelos AMMI com mais 
acurácia $\left(\mathrm{F}_{\mathrm{GH} 1} \mathrm{eF}_{\mathrm{GH} 2}\right)$ do que o teste de Gollob (1968). Mandel (1971) também considerou este teste como sendo bastante liberal, podendo resultar em falsos resultados de significância e provavelmente seleção de maior número de eixos, aumentando-se, assim, a ocorrência do erro tipo I.

Quanto à interpretação da porcentagem da $\mathrm{SQ}_{\mathrm{GxA}}$ original explicada pelo primeiro eixo AMMI, ressalta-se que não se deve proceder da maneira convencional, isto é, buscando-se uma explicação máxima dessa soma de quadrados. Conforme Gauch (1988), os primeiros eixos AMMI captam maior porcentagem de "padrão" e, com subseqüente acumulação de dimensões (eixos), ocorre diminuição na porcentagem de "padrão" e um acréscimo de "ruídos". Com isso, apesar da seleção de um só eixo, com pequena porção da $\mathrm{SQ}_{\mathrm{GxA}}$ original, espera-se estar captando maior porcentagem do "padrão". Por conseguinte, infere-se que os $64 \%$ restantes da $\mathrm{SQ}_{\mathrm{GxA}}$ provavelmente contenham pouca informação relevante, isto é, grande porção de "ruídos". Resultados semelhantes foram observados por Crossa et al. (1991), 27,1\%; Flores et al. (1996), 54,6\%; Pereira \& Costa (1998), 44,6\%.

Portanto, baixas proporções da $\mathrm{SQ}_{\mathrm{GxA}}$ sugerem contaminação da matriz de interações originais por fatores imprevisíveis, depreciando a qualidade de suas estimativas $(\hat{\mathrm{gaa}})_{\mathrm{ij}}$. Assim, pode-se inferir que, nestas condições, métodos tradicionais podem estar fornecendo predições de rendimentos de qualidade inferior às do modelo AMMI.

A Figura 1 mostra a representação biplot resultante da análise pelo modelo AMMI1. A abscissa representa os efeitos principais (médias de genótipos e de ambientes) e a ordenada, o primeiro eixo de interação (IPCA1). Assim, genótipos com valores de IPCA1 próximos a zero demonstram estabilidade aos ambientes de teste; combinações de genótipos e ambientes com escores IPCA de mesmo sinal têm interações específicas positivas; combinações de sinais opostos apresentam interações específicas negativas.

O genótipo que mais contribuiu para a interação GxA foi a cultivar FT-2000, pois apresentou a maior magnitude de escore no eixo de interação (Figura 1). Além disso, mostrou adaptabilidade específica ao local Jataí (JAT1 a JAT4), o que atesta sua instabilidade. Por sua vez, os genótipos que menos contribuíram para a interação GxA (mais estáveis) foram: L-112, L-21, L-11, L-9, L-16, L-13 e L-14 (menores coordenadas para o eixo IPCA1). Entre estes, destacaram-se as linhagens L-11, L-9, L-16, L-13 e L-14 por terem apresentado rendimentos acima da média geral. Assim, demonstram adaptabilidade geral, embora com respostas esperadas superiores nos cultivos de Goiânia, na segunda e na quarta época de semeadura (pontos relativos aos genótipos mais próximos a GO4e GO2).

Como, para fins de recomendação de cultivares, genótipos estáveis devem também apresentar desempenho desejável, o que é avaliado por suas médias (abscissa), conclui-se que as linhagens L-16, L-13 e L-14 são as mais promissoras para esse fim. As cultivares testemunhas Conquista, Emgopa 315 e MSOY-8001 (CONQ, EMG e MSOY, respectivamente), embora tenham apresentado as maiores produtividades médias, não foram tão estáveis aos ambientes como a maioria das linhagens experimentais, haja vista terem mostrado adaptação especial às condições de Jataí. Esse comportamento pode ter sido causado pela alta infestação de percevejos que ocor-

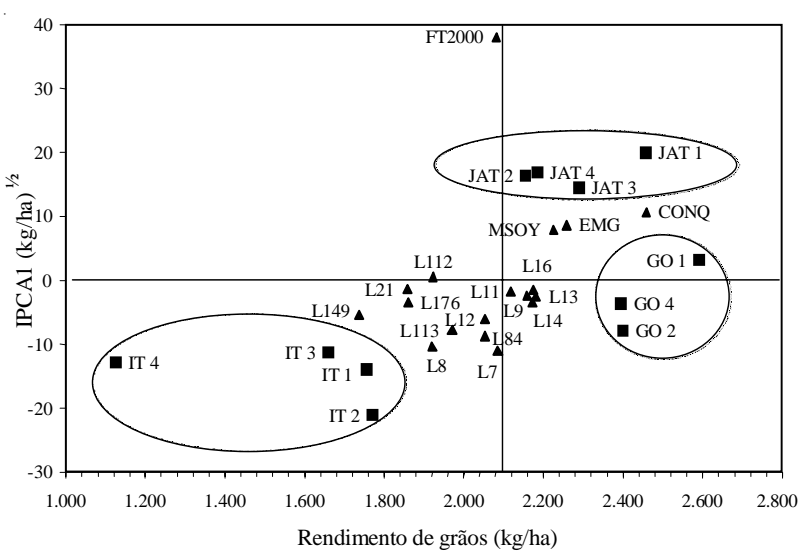

Figura 1. Biplot AMMI1 para dados de rendimento de grãos de soja, com 18 genótipos ( $\mathbf{\Delta}$ ) e 11 ambientes (ם) no Estado de Goiás (IT: Itumbiara; GO: Goiânia; JAT: Jataí). 
reu em alguns ambientes (IT1, IT2, IT3 e IT4) e pela superioridade genética das linhagens experimentais quanto à resistência a percevejos. Estas linhagens são oriundas de um programa de melhoramento com ênfase na resistência a insetos (uso do genitor resistente IAC-100), tendo manifestado claramente esta característica. Portanto, as linhagens experimentais mais promissoras apresentaram ampla adaptação a esse conjunto de ambientes.

Combinando-se as estimativas dos efeitos principais com a estimativa da interação revelada pelo padrão AMMI, pode-se ainda avaliar as respostas fenotípicas finais (produtividade) de cada genótipo em cada ambiente (Tabela 4). Os dados confirmam as adaptações específicas já discutidas, como a da cultivar FT-2000 ao local Jataí, e das linhagens L-13, L-14 e L-16 ao local Goiânia, assim como a má adaptação de certos genótipos a alguns ambientes, por exemplo, FT-2000 ao local Itumbiara e as linhagens L-149 e L-8 ao local Jataí.
Quanto aos ambientes, o local Itumbiara, na segunda época de semeadura (IT2), foi o que mais contribuiu para a interação GxA, pois apresentou o maior escore ambiental no eixo de interação (Figura 1). Por sua vez, em maior estabilidade destacou-se o local Goiânia (GO1, GO2 e GO4), com escores IPCA1 de menor magnitude. A inferioridade produtiva do ambiente IT4 foi causada por uma alta infestação de percevejos e déficit hídrico no período de enchimento de grãos.

A estabilidade ambiental informa sobre a confiabilidade no ordenamento dos genótipos num dado ambiente de teste, em relação à classificação para a média dos ambientes testados. Neste contexto, a maior estabilidade do local Goiânia, em relação a Itumbiara e Jataí, sugere que a classificação dos genótipos no primeiro local deve apresentar menor discordância em relação à classificação média em todos os locais. Portanto, o teste de materiais genéticos em Goiânia deve produzir ordenamento mais consistente e confiável para fins de recomendação de cultivares.

Tabela 4. Matriz de respostas preditas AMMI1 para dados de rendimentos de grãos (kg/ha), em soja, com 18 genótipos e 11 ambientes.

\begin{tabular}{|c|c|c|c|c|c|c|c|c|c|c|c|}
\hline \multirow[t]{2}{*}{ Genótipo } & \multicolumn{11}{|c|}{ Ambiente $^{(1)}$} \\
\hline & IT1 & IT2 & IT3 & IT4 & GO1 & $\mathrm{GO} 2$ & GO4 & JAT1 & JAT2 & JAT3 & JAT4 \\
\hline Conquista & 1.993 & 1.933 & 1.925 & 1.374 & 3.012 & 2.702 & 2.743 & 3.056 & 2.716 & 2.830 & 2.751 \\
\hline Emgopa 315 & 1.823 & 1.777 & 1.749 & 1.202 & 2.806 & 2.519 & 2.551 & 2.815 & 2.483 & 2.600 & 2.517 \\
\hline FT-2000 & 1.235 & 982 & 1.239 & 647 & 2.722 & 2.110 & 2.266 & 3.225 & 2.787 & 2.849 & 2.835 \\
\hline MSOY-8001 & 1.800 & 1.759 & 1.724 & 1.178 & 2.772 & 2.492 & 2.521 & 2.769 & 2.439 & 2.558 & 2.473 \\
\hline L-7 & 1.924 & 2.016 & 1.798 & 1.281 & 2.572 & 2.501 & 2.450 & 2.251 & 1.990 & 2.144 & 2.014 \\
\hline L-8 & 1.751 & 1.838 & 1.626 & 1.108 & 2.409 & 2.332 & 2.283 & 2.099 & 1.836 & 1.989 & 1.860 \\
\hline L-9 & 1.877 & 1.909 & 1.774 & 1.244 & 2.673 & 2.507 & 2.492 & 2.497 & 2.205 & 2.343 & 2.233 \\
\hline L-11 & 1.826 & 1.853 & 1.725 & 1.193 & 2.633 & 2.460 & 2.448 & 2.468 & 2.173 & 2.310 & 2.202 \\
\hline L-112 & 1.600 & 1.611 & 1.504 & 970 & 2.445 & 2.247 & 2.245 & 2.318 & 2.015 & 2.148 & 2.045 \\
\hline L-113 & 1.764 & 1.833 & 1.646 & 1.124 & 2.467 & 2.361 & 2.323 & 2.201 & 1.928 & 2.076 & 1.954 \\
\hline L-12 & 1.823 & 1.880 & 1.709 & 1.185 & 2.555 & 2.430 & 2.400 & 2.318 & 2.039 & 2.184 & 2.065 \\
\hline L-13 & 1.902 & 1.935 & 1.798 & 1.268 & 2.694 & 2.530 & 2.515 & 2.516 & 2.224 & 2.362 & 2.252 \\
\hline L-14 & 1.905 & 1.944 & 1.799 & 1.270 & 2.682 & 2.528 & 2.509 & 2.489 & 2.200 & 2.340 & 2.228 \\
\hline L-149 & 1.496 & 1.549 & 1.385 & 859 & 2.240 & 2.108 & 2.080 & 2.014 & 1.733 & 1.876 & 1.759 \\
\hline L-16 & 1.879 & 1.904 & 1.778 & 1.247 & 2.690 & 2.514 & 2.503 & 2.529 & 2.234 & 2.370 & 2.263 \\
\hline L-176 & 1.593 & 1.632 & 1.486 & 958 & 2.370 & 2.216 & 2.197 & 2.177 & 1.888 & 2.028 & 1.916 \\
\hline L-21 & 1.561 & 1.586 & 1.461 & 929 & 2.375 & 2.197 & 2.187 & 2.217 & 1.921 & 2.057 & 1.949 \\
\hline$\underline{\mathrm{L}-84}$ & 1.860 & 1.936 & 1.739 & 1.219 & 2.547 & 2.451 & 2.409 & 2.264 & 1.995 & 2.145 & 2.020 \\
\hline $\mathrm{CV}(\%)$ & 15,30 & 20,10 & 15,79 & 25,14 & 20,68 & 20,16 & 17,00 & 9,94 & 11,27 & 15,99 & 11,73 \\
\hline
\end{tabular}

${ }^{(1)}$ Locais: Itumbiara (IT), Goiânia (GO) e Jataí (JAT); os números 1, 2, 3 e 4 após a sigla do local indicam as épocas de semeadura, respectivamente 28/nov., 4/dez., 11/dez. e 18/dez. 


\section{Conclusões}

1. A análise AMMI apresenta melhor qualidade nas predições de respostas fenotípicas do que os métodos tradicionais, sobretudo quando o padrão da interação GxA é captado apenas pelo primeiro termo multiplicativo da análise.

2. As linhagens experimentais são relativamente mais estáveis do que as cultivares testemunhas, e estas, embora mais produtivas, são adaptadas às melhores condições ambientais (local Jataí).

3. As linhagens L-16, L-13 e L-14 apresentam estabilidade agronômica, sendo, portanto, as mais promissoras para uma recomendação geral de cultivares; já as linhagens L-112 e L-21, também estáveis em comportamento, são melhor adaptadas ao local Goiânia.

4. O local Goiânia é mais indicado para o teste de linhagens do que Jataí e Itumbiara.

\section{Agradecimentos}

Ao professor Lázaro Chaves, pelas sugestões e incentivo; aos colegas de pós-graduação Edson de Oliveira e Américo Nunes da Silveira, pela colaboração e apoio na condução dos experimentos e coletas de dados; à Universidade Federal de Goiás, pela oportunidade de realização do curso; e à Capes, pela concessão da bolsa de estudos.

\section{Referências}

COCHRAN, W. G. The combination of estimates from different experiments. Biometrics, Washington, v. 10, p. 101-129, 1954.

CORNELIUS, P. L.; SEYEDSADR, M.; CROSSA, J. Using the shifted multiplicative model to search for "separability" in crop cultivar trials. Theoretical and Applied Genetics, Berlin, v. 84, p. 161-172, 1992.

CROSSA， J.; FOX, P. N.; PFEIFFER, W. H.; RAJARAM, S.; GAUCH, H. G. AMMI adjustment for statistical analysis of an international wheat yield trial. Theoretical and Applied Genetics, Berlin, v. 81, p. 27-37, 1991.

CRUZ, C. D.; TORRES, R. A. de A.; VENCOVSKY, R. An alternative approach to the stability analysis proposed by Silva and Barreto. Revista Brasileira de Genética, Ribeirão Preto, v. 12, n. 3, p. 576-580, 1989.
DUARTE, J. B.; VENCOVSKY, R. Interação genótipos x ambientes: uma introdução à análise AMMI. Ribeirão Preto: Sociedade Brasileira de Genética, 1999. 60 p. (Série Monográfica, 9).

DUARTE, J. B.; ZIMMERMAN, M. J. de O. Adaptabilidade e estabilidade de rendimento de genótipos de feijão comum. Pesquisa Agropecuária Brasileira, Brasília, v. 29, n. 1, p. 25-32, jan. 1994.

EBERHART, S. A.; RUSSELL, W. A. Stability parameters for comparing varieties. Crop Science, Mandison, v. 6, p. 36-40, 1966.

EMBRAPA. Centro Nacional de Pesquisa de Soja (Londrina, PR). Recomendações técnicas para a cultura da soja na região central do Brasil. Londrina, 2000. 226 p. (Documentos, 132).

FLORES, F.; MORENO, M. T.; CUBERO, J. I. Genotype-environment interaction in faba bean: comparison of AMMI and principal coordinate model. Field Crops Research, Amsterdam, v. 47, p. 117-127, 1996.

GABRIEL, K. R. The biplot graphic of matices with application to principal component analysis. Biometrika, London, v. 58, n. 3, p. 453-467, 1971.

GAUCH, H. G. Model selection and validation for yield trials with interaction. Biometrics, Washington, v. 44, p. 705-715, 1988.

GAUCH, H. G.; ZOBEL, R. W. AMMI analysis of yield trials. In: KANG, M. S.; GAUCH, H. G. (Ed.). Genotype by environment interaction. Boca Raton: CRC Press, 1996. v. 4, p. 85-122.

GOLLOB, H. F. A statistical model which combines features of factor analytic and analysis of variance techniques. Psychometrika, Toronto, v. 33, n. 1, p. 73-145, Jan. 1968.

HIROTSU, C. An approach to defining the pattern of interaction effects in a two-way layout. Annals of the Institute of Statistical Mathematics, Dordrecht, v. 35, p. 77-90, 1983.

KANG, M. S. Using genotype-by-environment interaction for crop cultivar development. Advances in Agronomy, San Diego, v. 62, p. 199-252, 1998.

KEMPTON, R. A. The use of biplots in interpreting variety by environment interactions. Journal of 
Agricultural Science, Cambridge, Inglaterra, v. 103, p. 123-135, 1984.

MANDEL, J. A new analysis of variance model for non-additive data. Technometrics, Alexandria, v. 13, p. 1-18, 1971.

PEREIRA, A. S.; COSTA, M. D. Análise de estabilidade de produção de genótipos de batata no Rio Grande do Sul. Pesquisa Agropecuária Brasileira, Brasília, v. 33, n. 4, p. 405-409, abr. 1998.

PIEPHO, H. P. Best linear unbiased prediction (BLUP) for regional yield trials: a comparison to additive main effects and multiplicative interaction (AMMI) analysis. Theoretical and Applied Genetics, Berlin, v. 89, p. 647-654, 1994.

PIEPHO, H. P. Robustness of statistical test for multiplicative terms in the additive main effects and multiplicative interaction model for cultivar trial. Theoretical Applied of Genetics, Berlin, v. 90, p. 438-443, 1995.

RAMALHO, M. A. P.; FERREIRA, D. F.; OLIVEIRA, A. C. Experimentação em genética e melhoramento de plantas. Lavras: Ufla, 2000. 326 p.

SEDIYAMA, C. S.; OLIVEIRA, L. O.; CRUZ, C. D. Análise de estabilidade fenotípica de cultivares de soja por meio da regressão linear simples e da regressão linear segmentada. Revista Ceres, Viçosa, MG, v. 37, p. 513-518, 1990.

SILVA, J. G. C. Análise da adaptabilidade através de regressão linear segmentada. 1. Fundamentos. Pesquisa Agropecuária Brasileira, Brasília, v. 30, n. 4, p. 435-448, 1995.

ZOBEL, R. W.; WRIGHT, M. J.; GAUCH, H. G. Statistical analysis of a yield trial. Agronomy Journal, Madison, v. 80, p. 388-393, 1988. 